\title{
Constraints on warm power-law inflation in light of Planck results
}

\author{
Zahra Ghadiri, ${ }^{*}$ Ali Aghamohammadi, ${ }^{\dagger}$ and Abdollah Refaei ${ }^{\ddagger}$
}

Sanandaj Branch, Islamic Azad university, Sanandaj, Iran

\author{
Haidar Sheikhahmadi ${ }^{\S}$ \\ School of Astronomy, Institute for Research in Fundamental Sciences (IPM), \\ P. O. Box 19395-5531, Tehran, Iran and \\ Center for Space Research, North-West University, Mafikeng, South Africa
}

\begin{abstract}
The constraints on a general form of the power-law potential and the dissipation coefficient in the framework of warm single field inflation imposed by Planck data will be investigated. By Considering a quasi-static Universe, besides a slow-roll condition, the suitable regions in which a pair of theoretical free parameters are in good agreement with Planck results will be estimated. In this method instead of a set of free parameters we can visualize a region of free parameters that can satisfy the precision limits on theoretical results. On the other side, when we consider the preformed quantity for the amplitude of scalar perturbations, the conflict between obtained results for free parameters in different steps dramatically will be decreased. As have done in prominent literature, based on the friction of the environment, we can divide the primordial Universe to the two different epochs namely weak and strong dissipative regimes. For the aforementioned eras, the free parameters of the model will be constrained and the best regions will be obtained. To do so, the main inflationary observables such as tensor-to-scalar ratio, power-spectra of density perturbations and gravitational waves, scalar and tensor spectral indices, running spectral index and the number of e-folds in both weak and strong regimes will be obtained. Ultimately, it can be visualized, this model can make concord between theoretical results and data originated from cosmic microwave background and Planck 2013, 2015 and 2018.
\end{abstract}

PACS numbers: 98.80.Cq; 04.20.CV

keywords:Warm power-law inflation, Weak and strong dissipative regimes, Planck 2013 and 2015 


\section{INTRODUCTION}

In this work by virtue of the Planck results [1-5] the free parameters of both the power-law potential and the dissipation coefficient in the framework of warm inflation will be constrained.

After four decades of introducing, now the inflation can be considered as the most acceptable paradigm to explain the evolution of early Universe [6-12]. The main achievement of this theory goes back to cope with three well-known problems of the standard big bang theory namely the horizon, flatness and monopoles problems [13-18]. Even better, the inflation theory provides a mechanism to explain structure formation and the source of the observed anisotropies in the Cosmic Microwave Background (CMB) radiation [19-21]. Besides, this scenario could assess a correct result for the amplitude of primordial perturbations compared to observations [14, 22-31]. The various inflationary models have been investigated in $[13,32]$, that could be divided into two well-known approaches as super-cold and warm inflationary proposals. In the warm inflation scenario [33-40], during the initial rapid expansion of the Universe, the continuous radiation production based on thermal perturbations is arisen and so the interaction between radiation and other components of the extra hot primordial soup can be visualized [34,35]. One important result from such interactions is that the energy density evolutions could be considered almost constant [33]. Thanks to such evolutions the inflationary phase smoothly enteres into radiation epoch, without introducing pre-heating and reheating phases which are critical parts of super-cold inflation models. In other word, The remedy of the warm inflation to get rid of the graceful exit problem which super-cold inflation was faced, could be considered as an advantage, for more details we refer the reader to $[35,41-48]$. One way to show the interaction between different components of the primordial thermal bath, i.e. inflationary era, is invoking the dissipation effect as a rule of the flow of energy between them [49]. Consequently, such interactions cause a high friction environment and the friction term, i.e. $\Gamma \dot{\phi}^{2}$, that will be appeared in the conser-

\footnotetext{
*Electronic address: zghadiry@gmail.com

†Electronic address: a.aqamohamadi@gmail.com;a.aghamohamadi@iausdj.ac.ir

‡Electronic address: abr412@gmail.com;A.Refaei@iausdj.ac.ir

§Electronic address: h.sh.ahmadi@gmail.com;h.sheikhahmadi@ipm.ir
} 
vation equations of scalar field and radiation, for a precision study one can see [50]. As a supplementary discussion, a principal condition in order to happen the warm inflation is that the radiation temperature satisfy condition $T>H$, where $T$ and $H$ are temperature and Hubble parameter respectively [51-53]. In Refs. [42-45], it was noticed that the quantum fluctuation and thermal perturbations, could be dependent upon the parameters $T$ and $H$ respectively. According to the condition $T>H$, the thermal fluctuations play a crucial role in producing the primary density perturbations, as a seeds for large-scale structure formation. In this case, the thermal fluctuations entirely dominated in comparison to the quantum portion in primordial fluctuations [43-45, 54].

On the other hand, in 1983, A. Linde has introduced a new proposal for inflation based on the chaotic scalar field namely chaotic inflation [9]. In this theory he put forward the power law potential, as same as the quantum field theory, and it plays the role of corner stone of his theory. Hence the power law potentials received a lot of interests, because of their simplicity and compatibility with observations and also solving the graceful exit problem of old and new inflation. But the answer of this simple query that, which exponents are able to run warm inflation in a better way compared to Planck results is remained ambiguous [24]. Therefore our main motivation to start this study is finding the answers of the aforementioned question. Technically, for both regimes we will calculate the regions which give the best estimated values for free parameters of our model. After that, we will examine the accuracy of our estimations for some pares of best fitted parameters compared to our criterion, i.e. Planck 2013, 2015 and 2018 constraints on inflationary data [1-5].

This paper is organized as follows: In Section 2, the main dynamical equations of warm inflation will be expressed and so the inflationary parameters will be evaluated. Additionally, Section 3 will be devoted to study on the power-law potentials in a weak dissipative regime and the region which contains the best fitted free parameters of the model will be appeared. And Section 4 will be dedicated to investigate the behaviour of power-law potentials in the strong dissipative regime. Similar to the weak regime, the parameters which can derive warm inflation will be estimated by virtue of the Planck results. Ultimately, Section 5 is devoted to conclusion and final remarks. 


\section{GENERAL FRAMEWORK}

There is a spatially flat Friedmann-Limaitre-Robertson-Walker (FLRW) space time with signature -2 . We assume that the Universe consists of two different components, in which one of them can be a self-interacting scalar field $\phi$ and all the remnant components are considered as perfect fluid as well. The energy density and pressure of such scalar field are expressed as follows:, respectively,

$$
\rho_{\phi}=\frac{\dot{\phi}^{2}}{2}+V(\phi)
$$

and

$$
P_{\phi}=\frac{\dot{\phi}^{2}}{2}-V(\phi)
$$

The energy density of the aforementioned perfect fluid, i.e. almost radiation, is presented as $\rho_{r}$. Also the first Friedmann equation could be expressed as follows:

$$
H^{2}=\frac{1}{3 M_{p}^{2}}\left(\rho_{\phi}+\rho_{r}\right),
$$

where $M_{p}^{2}=\frac{1}{8 \pi G}$ is the well-known reduced Planck mass and is of the order of $10^{18} \mathrm{GeV}$. The evolution equation of scalar field in warm inflation can be expressed as $[43-45,54,55]$

$$
\ddot{\phi}+3 H(1+Q) \dot{\phi}+V^{\prime}=0
$$

where the parameter $Q \equiv \Gamma / 3 H$ is introduced as an anomalous dissipation function, that should be determined, and $\Gamma$ is the dissipation coefficient, usually can be introduced as an ansatz. The conservation equations of $\rho_{\phi}$ and $\rho_{r}$ are described by the following equations $[42,54]$

$$
\dot{\rho_{\phi}}+3 H\left(\rho_{\phi}+P_{\phi}\right)=-\Gamma \dot{\phi}^{2}
$$

and

$$
\dot{\rho}_{r}+4 H \rho_{r}=\Gamma \dot{\phi}^{2}
$$

where the dissipation coefficient is a positive parameter, $\Gamma>0$. From above equations it is understood that the flow of energy is from scalar field to the radiation. We can consider an ansatz for dissipation coefficient $\Gamma(T, \phi)$ as

$$
\Gamma(T, \phi)=a T^{n} \phi^{1-n},
$$


here the parameter $T$ is the temperature of the fluid and for the certain reasons the dissipation coefficient can be introduced as a function of temperature and scalar field [42, 56-59, 69]. In fact, the main motivation is taken from [53, 57-60,67,68], in which $n=1$ correspond to $\Gamma \propto T$, that is describing the high-temperature super symmetry case; for $n=0$, the $\Gamma$ is only depends on the scalar field, $\Gamma \propto \phi$ which imparts an exponentially decaying propagator in the super symmetry case; and for $n=-1$, there is $\Gamma \propto T^{-1} \phi^{2}$, which is consistent with the non-supersymmetry case. As well, the case $n=3, \Gamma=T^{3} \phi^{-2}$ was investigated in [68]. During warm inflation, the energy density of scalar field is the dominant component in comparison to the radiation one i.e. $\rho_{\phi} \gg \rho_{r}$ [42-45]. In other words, the expansion rate is smaller than the radiation energy density, i.e. $\rho_{r}^{1 / 4}>H$ or $T>H$, which is a critical requirement of happening a healthy warm inflation. Besides, if we consider the notion of the slow roll conditions, it is assumed that during inflation the radiation production becomes quasi-stable i.e. $\quad \dot{\rho}_{r} \ll 4 H \rho_{r}, \Gamma \dot{\phi}^{2}[42,43,45]$ and according to (2) one concludes $\ddot{\phi} \ll 3 H(1+Q) \dot{\phi}$. Thence, the equations (2) and (4) can be approximated as

$$
\begin{aligned}
\rho_{r}=\alpha T^{4} & \simeq \frac{\Gamma \dot{\phi}^{2}}{4 H}, \\
3 H(1+Q) \dot{\phi} & \simeq-V_{, \phi} .
\end{aligned}
$$

where $\alpha=\pi^{2} g_{\star} / 30$ is the Stefan-Boltzmann constant, that, the number of degrees of freedom of the radiation field is $g_{\star}=228.75$ in the Minimal Supersymmetric Standard Model (MSSM), hence for the parameter $\alpha$ we receive $\alpha=70$. The necessary condition for inflation in the warm inflation context, is that the slow-roll parameters Eq.(8) obey Eq.(9)

$$
\epsilon=\frac{M_{p}^{2}}{2}\left(\frac{V_{, \phi}}{V}\right)^{2}, \eta=M_{p}^{2}\left(\frac{V_{, \phi \phi}}{V}\right)
$$

where the slow-roll conditions for warm inflation must satisfy the following conditions [ 45 , $60-65]$

$$
\epsilon \ll 1+Q, \quad \eta \ll 1+Q .
$$

Based on the definitions of slow-roll parameters whenever one of these parameters becomes equal to $1+Q$ the inflation process is terminated. Another important parameter to describe the inflationary evolution is the number of e-folds. This parameter plays an important role in solving the horizon problem and is defined as

$$
N=-\int_{\phi_{\text {end }}}^{\phi} \frac{H}{\dot{\phi}} d \phi
$$


Additionally, to estimate, best values for, the free parameters of the model we have to obtain the amplitude of scalar and tensor perturbations, tensor-to-scalar spectrum ratio, the scalar spectral index and running parameter which are expressed respectively as follows. Before going forward, we should emphasise we considered a much more accepted expression for the amplitude of the primordial spectrum [70, 71].

$$
\begin{gathered}
\mathcal{P}_{s}=\frac{25}{4} \frac{H^{2}}{\dot{\phi}^{2}} \delta \phi^{2}, \\
\mathcal{P}_{t}=\frac{2 H^{2}}{\pi^{2} M_{p}^{2}}, \\
r \equiv \frac{\mathcal{P}_{t}}{\mathcal{P}_{s}}, \\
n_{s}-1 \equiv \frac{d \ln \mathcal{P}_{s}}{d \ln k},
\end{gathered}
$$

and

$$
\alpha_{s}=\frac{d n_{s}}{d \ln k}
$$

From the above equations and slow roll conditions it is understood that at the sound horizon exit, i.e. $a H=c_{s} k$, one can obtain [66]

$$
\frac{\mathrm{d}}{\mathrm{dlnk}} \simeq-\frac{\mathrm{d}}{\mathrm{dN}}
$$

in above relation, cause of slow roll notion, the parameters $H$ and sound speed $c_{s}$ are considered as constant.

In the following, the calculations will be divided into two different sections namely the weak and strong dissipation regimes, i.e. $Q \ll 1$ and $Q \gg 1$ respectively. Because of the importance of power-law potentials in deriving inflation we are going to consider a general form of the power-law potentials and investigate the behaviour of such potentials in the framework of warm inflation scenario. In short, for the weak dissipation regime we shall see that the parameter which drives inflation is the Hubble parameter. But in the strong regime, the situation because of the high friction environment, is completely different and the evolution of inflation is operated by the dissipation coefficient $\Gamma$. The impact of different types of dissipation coefficients were investigated extensively in the literature and we refer the reader to $[51,58,59]$. For both regimes we interested in finding the best fitted free parameters of the model especially for the exponents of variables of potential and dissipation coefficient functions. 


\section{CONSISTENCY OF POWER-LAW POTENTIAL IN WEAK DISSIPATIVE REGIME}

In this section, given that in this section our model evaluates based on the weak dissipation regime in which $Q \ll 1$, by introducing the potential as

$$
V(\phi)=V_{0} \phi^{k}
$$

and imposition slow-roll approximations, the Eqs. (1) and (2) take the following form

$$
H^{2}=\frac{1}{3 M_{p}^{2}} V(\phi)
$$

and

$$
3 H \dot{\phi}+V_{, \phi}=0
$$

where subscript $(, \phi)$ stands for derivative with respect to $\phi$. In Eq.(6) the temperature of the radiation according to the Eqs. (5) and (17) obtained as

$$
T=\gamma \phi^{\frac{2-k+2 n}{2(-4+n)}},
$$

where $\gamma=\left(\frac{a k^{2} M_{p}{ }^{3} \sqrt{V_{0}}}{36 \alpha}\right)^{\frac{1}{4-n}}$. In addition, the first slow-roll parameter (8) for this regime reduces to

$$
\epsilon=\frac{k^{2} M_{p}^{2}}{2 \phi^{2}}
$$

Generally, the inflation period terminates when $\epsilon=1$, where using Eq.(21), one achieves

$\phi_{\text {end }}=\frac{k M_{p}}{\sqrt{2}}$. Besides, from Eq.(10) the value of scalar field at the time of the exit of the horizon is given by the following equation

$$
\phi=\sqrt{2 k M_{p}^{2} N+\phi_{\text {end }}^{2}} .
$$

In the following, we want to calculate the perturbation parameters based on our model and compare them with observational data. To do so, we consider Eq.(11) in which for weak regime leads $\delta \phi^{2} \simeq H T$. Whereas the fluctuations in the warm inflationary theory are created by the thermal fluctuations instead of the quantum fluctuations, [51], accordingly the amplitude of scalar perturbation becomes

$$
\mathcal{P}_{s}=\frac{25 \gamma}{4 k^{2} M_{p}{ }^{5}}\left(\frac{k^{2} M_{p}{ }^{2}}{2}+2 k M_{p}{ }^{2} N\right)^{\frac{14+5 k-n k-6 n}{4(4-n)}} \sqrt{\frac{\mathrm{V}_{0}}{3}} .
$$


From Eq.(23) and definition of $\gamma$ we can express $a(n, k, N)$ as follows:

$$
\begin{aligned}
a(n, k, N) & =\left(\sqrt{\frac{3}{V_{0}}} \frac{4 k^{2} M_{p}^{5}}{25 \gamma^{*}}\right)^{4-n} \\
& \times\left(\frac{k^{2} M_{p}^{2}+4 k M_{p}^{2} N}{2}\right)^{\frac{(14+5 k-n k-6 n)}{-4}} \mathcal{P}_{s}^{4-n}
\end{aligned}
$$

Here $\gamma^{*}=\left(\frac{k^{2} M_{p}{ }^{3} \sqrt{V_{0}}}{36 \alpha}\right)^{\frac{1}{4-n}}$ is introduced for more convenience in writing the equations and we shall consider the observational value for the amplitude of scalar perturbation as $2.17 \times 10^{-9}$. Sometimes although the free parameters satisfy the observational constraints on quantity the scalar spectral index, it could not concluded that they can make a concord between observations and theoretical results for $\mathcal{P}_{s}$. So it is better we examine the accuracy of the free parameters of the model based on Planck results as our criterion. For instance here we can introduce our free parameters of the model as $n, k, N$ and other parameters can be estimated based on the behaviour of these parameters as well. To do so, at first, from Eqs.(14) and (15) the scalar spectral index and its running could be achieved as

$$
n_{s}(n, k, N)=\frac{14+k-6 n-16 N+4 n N}{(-4+n)(k+4 N)} .
$$

and

$$
\alpha_{s}(n, k, N)=-\frac{4(-14+k(-5+n)+6 n)}{(-4+n)(k+4 N)^{2}} .
$$

Also from Eq.(12), the tensor power spectrum in terms of the number of e-fold is obtained as

$$
\mathcal{P}_{t}=\frac{2\left(\frac{k^{2} M_{p}{ }^{2}}{2}+2 k M_{p}^{2} N\right)^{k / 2} V_{0}}{3 M_{p}^{4} \pi^{2}} .
$$

Additionally, from Eq. (13), the tensor to scalar ratio is obtained as

$$
\begin{aligned}
r(n, k, N) & =\sqrt{\frac{\mathrm{V}_{0}}{3}} \\
& \times \frac{\left(\frac{k^{2} M_{p}^{2}+4 k M_{p}^{2} N}{2}\right)^{\frac{3 k+6 n-k n-14}{2(8-2 n)}}}{25\left(8 k^{2} M_{p}\right)^{-1} \pi^{2} \gamma^{*}} a^{\frac{1}{n-4}} .
\end{aligned}
$$

Now to make the constraints on free parameters of the model, we need to compare them with observational data originated from Planck 2013 and 2015 data [1, 2, 4]. For this purpose, we plot the free parameters $n-k$ diagram by virtue of Eqs.(28) and (25) for the Confidence Levels (CLs) 68\% and 95\% CL allowed by Planck 2015, TT, TE, EE+lowP data [4]. This figure 1 shows the regions in which the pair of free parameters, they can make a set of $n, k$, satisfy the constraints originated from Planck data. 


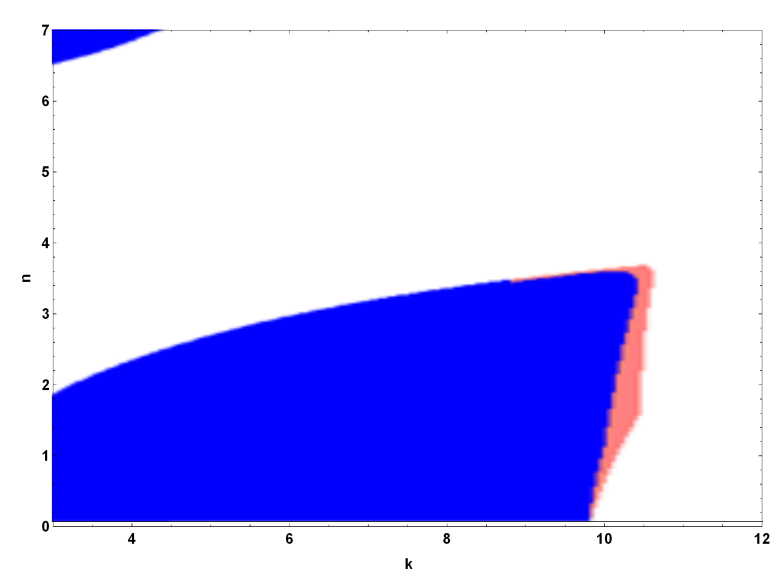

FIG. 1: This figure shows a set of pair $(n, k)$ that can give the best fitted values based on the $\left(r-n_{s}\right)$ diagram originated from Planck 2015 observational data. Here the dark blue color shows the results for 68\% CL of Planck 2015 data, and the light red color shows those, that put the result in 95\% $C L$. To plot this diagram we used $V_{0}=2.7 \times 10^{-25}$, and $M_{p}=1$..

Now by means of an arbitrary set of the aforementioned pairs we can re-plot the $r-n_{s}$ diagram as one of the most important instrument to test the accuracy of a theoretical model. So the figure 2 shows the diagram $r-n_{s}$ based on different types of data mentioned in the caption. Figure 2 indicates that our results are in a good agreement compared to the observations.

The latest observational data suggest that the amplitude of scalar perturbation at the horizon crossing is very close to the amount of $\mathcal{P}_{s}=2.17 \pm 0.1 \times 10^{-9}$, and the tensor-toscalar ratio has an upper limit as $r<0.11$ at $68 \%$ [4]. In what following, by means of Eqs.(26) and (25) we want to plot the diagram of running parameter, $d n_{s} / d N-n_{s}$ and then compare the obtained results with the observations originated from Planck data. Figure 3 which indicates the prediction of this model can lie inside the joint 68\% CL region of Planck 2015 TT, TE, EE+lowP data [4, 5], and so could satisfy the compatibility with observations.

An important feature of the warm inflationary model is that the thermal fluctuations overcome the quantum fluctuations, since the fluid temperature is bigger than the Hubble parameter, i.e. $T>H$. To receive a healthy warm inflation, this condition should be justified during the cosmological evolution. Fig. 4 expresses the behavior of the ratio of the temperature to the Hubble parameter during such era. From the Figure 4, one can observe 

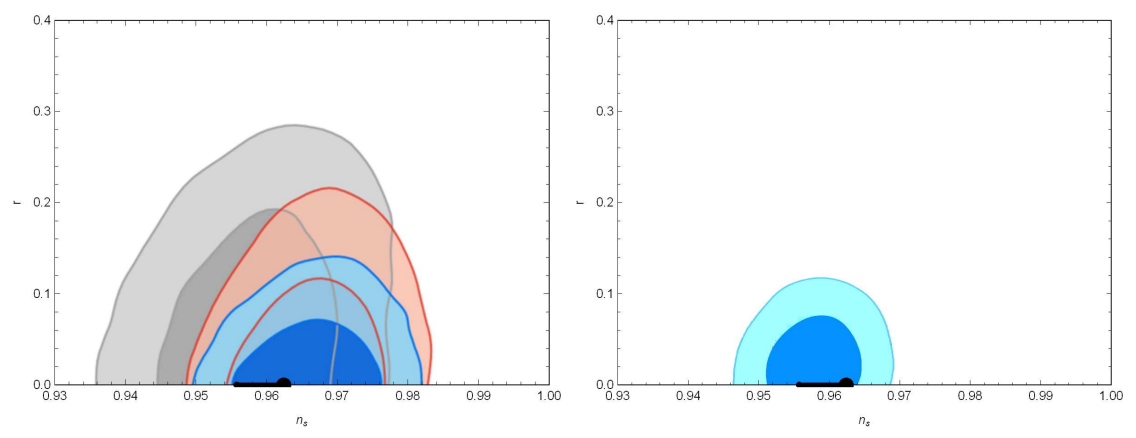

FIG. 2: The $r-n_{s}$ diagram shows Prediction of the model in the weak regime for free parameters $\alpha=70, k=6, n=2, V_{0}=2.7 \times 10^{-25}, M_{p}=1$ and $N_{e}=60$, in comparison to the observational data risen by Planck 2013, 2015 and 2018. In the left panel, the likelihood of Planck 2013 are indicated with grey contours, Planck 2015 TT+lowP with red contours, and Planck 2015 TT,TE,EE+lowP with blue contours. And in the right panel, the results of Planck 2018 are indicated by dark and light blue colours referring $68 \%$ and $95 \%$ confidence levels respectively. In both figures the thick black lines refers the predictions of theoretical results in which small and large circles are the values of $n_{s}$ at the number of e-folds $N=55, N=65$ respectively.

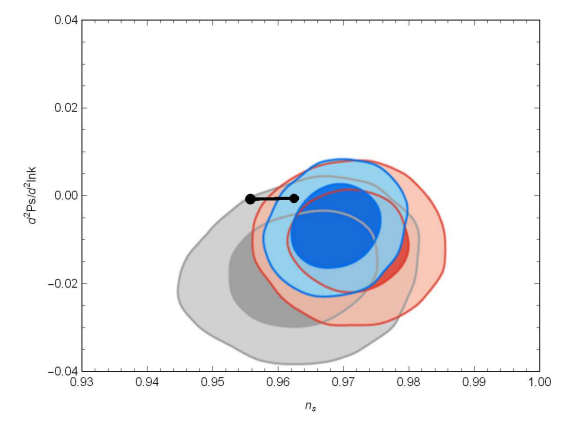

FIG. 3: In this figure the thick black line depicts the predictions of our model in which small and large circles are the value of $n_{s}$ at the number of e-fold $N=55, N=65$ respectively. To plot this shape we use the free parameters $k=6, n=2, V_{0}=2.7 \times 10^{-25}, M_{p}=1, N_{e}=65$ and $\alpha=70$. In this figure the grey contours indicates the likelihood of Planck 2013, Planck 2015 TT+lowP showed with red contours and the blue contours considered for Planck 2015 TT, TE,EE+lowP.

that the condition is satisfied during the inflationary phase of cosmological dynamics.

Although it has postulated that $Q<<1$ in the weak regime, it should be controlled by virtue of the best fitted free parameters of the model. Hence one can observe from Fig. 5 this condition is satisfied properly. 


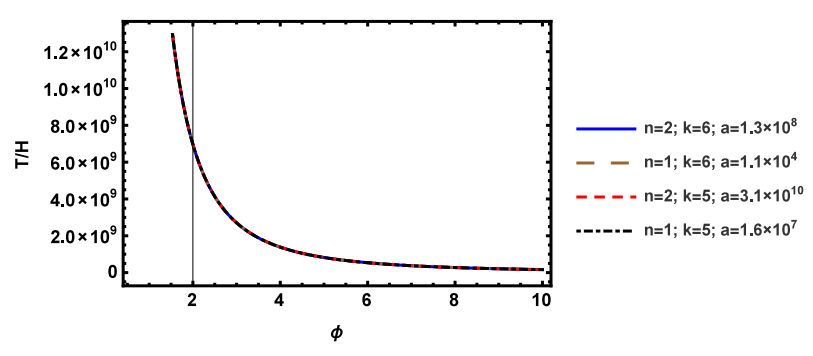

FIG. 4: The plot shows the ratio of the temperature to the Hubble parameter during the inflationary period of the model in the weak dissipative regime versus the inflaton scalar field, $\phi$, for different values of $(n, k)$ and the parameter $a$. As one can see from the plots during inflation the temperature is larger than the Hubble parameter, and the condition $T>H$ is satisfied properly. To draw these figures we fixed the value of $V_{0}$ at $10^{-25}$.

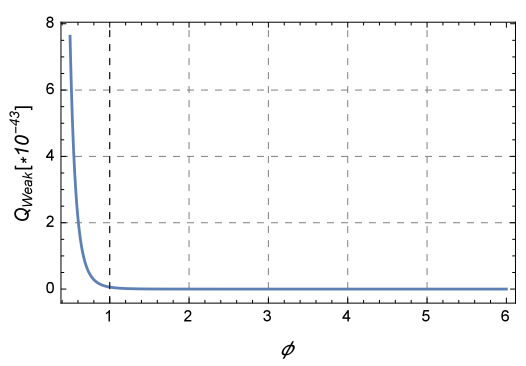

FIG. 5: This figure indicates the ratio of the dissipation parameter,, , to the Hubble parameter during the inflationary period of the model in the weak dissipative regime versus the inflaton scalar field, $\phi$, for the value of $(n=3, k=7)$ and the parameter $a=10^{-4}$. As observed from the plot during inflation the parameter $\Gamma$ is much less than the Hubble parameter, and the condition $Q=\Gamma / 3 H<<1$ is satisfied properly. To draw these figure we fixed the value of $V_{0}$ at $10^{-25}$.

\section{CONSISTENCY OF POWER-LAW POTENTIAL IN STRONG DISSIPATIVE REGIME}

Here we want to continue our calculations this time for strong dissipation regimes namely $Q \gg 1$. Clearly by considering Eqs. (1), (7), (17) and slow-roll approximation, the Friedman and evolution scalar field equations take the following form respectively,

$$
H^{2}=\frac{1}{3 M_{p}^{2}} V(\phi),
$$

and

$$
3 H Q \dot{\phi}+V_{, \phi}=0
$$


Using the Eqs. (5), (6), (30) and by virtue of the above inflationary potential, i.e. (17), the temperature could be obtained as follows:

$$
T=\bar{\gamma} \phi^{\frac{-3+\frac{3 k}{2}+n}{4+n}}
$$

where

$$
\bar{\gamma}=\left(\frac{k^{2} M_{p} \sqrt{V_{0}^{3}}}{2520 \alpha}\right)^{\frac{1}{4+n}} a^{\frac{-1}{4+n}}=\overline{\gamma^{*}} a^{\frac{-1}{4+n}}
$$

Now, by virtue of the above equations the first slow-roll parameter and dissipation term $Q$ are obtained respectively as bellow:

$$
\epsilon=\left(\frac{k M_{P}}{\sqrt{2} \phi}\right)^{2}
$$

and

$$
Q=\frac{M_{P} a \bar{\gamma}^{n}}{\sqrt{3 V_{0}}} \phi^{\frac{4-6 n-2 k+k n}{4+n}}
$$

For the strong regime usually inflation ends up when $\epsilon \approx Q$, so using Eq.(33) the scalar field related to such high friction eras is obtained as

$$
\phi_{\text {end }}=\left(\frac{2 a \bar{\gamma}^{n}}{M_{p} k^{2} \sqrt{3 V_{0}}}\right)^{-\frac{4+n}{12-2 k-4 n+k n}} .
$$

Additionally, from Eq.(10) the value of the scalar field based on the horizon line crossing for perturbations is given by

$$
\phi=\left(k M_{P} \sqrt{3 V_{0}} \bar{\gamma}^{(-n)} a^{-1}\left[(2+\lambda) N+\frac{k}{2}\right]\right)^{\frac{1}{2+\lambda}},
$$

where $\lambda=\frac{4-6 n-2 k+k n}{4+n}$. In this regime, similar to the weak case, to specify scalar power spectrum we consider the Eq.(11) but here $\delta \phi^{2} \simeq k_{F} T / 2 \pi^{2}$ where $k_{F}=\sqrt{\Gamma H}$ and it can be appeared as follows $[51,55]$ :

$$
\mathcal{P}_{s}=\frac{25}{32 \pi^{2}}\left(\frac{4 \Gamma^{3} V^{3 / 2}}{\alpha^{1 / 3} 3^{3 / 2} M_{P}{ }^{3} V^{\prime 2}}\right)^{\frac{3}{4}} .
$$

Now by substituting Eqs.(5, 31) and (35) into Eq.(36) one obtains

$$
\begin{aligned}
& \mathcal{P}_{s}=\frac{25\left(\frac{\left(\overline{\gamma^{*}}\right)^{3 n}}{k^{2} M_{P}^{3} \sqrt{V_{0}} \alpha^{1 / 3}}\right)^{\frac{3}{4}}}{3^{1 / 8} 24 \pi^{2} \sqrt{2}} \times \\
& \left(\left[\sqrt{3} k M_{P} \sqrt{V_{0}} \overline{\gamma^{*}}(-n)\left(N(2+\lambda)+\frac{k}{2}\right)\right]^{\frac{1}{2+\lambda}}\right)^{\frac{3 \beta}{4}} a^{3 \psi}
\end{aligned}
$$


where $\beta=\frac{-2 k+8+4 k n-3 n}{4+n}$ and

$$
\psi=\frac{-4 k-k n+15 n+24}{(-4+k) n^{2}+2 k n-8 k+4 n+48} .
$$

To make a better agreement between observational data and theoretical results of our investigation we can follow the approach which done in weak regime. Thence, if we want to precisely consider the Planck results for $\mathcal{P}_{s}$, the parameter $a$ based on $\mathcal{P}_{s}$ and the free parameters of the model can be achieved as

$$
\begin{aligned}
& a(n, k, N)=\left(\frac{3^{1 / 8} 24 \pi^{2} \sqrt{2}}{25\left(\frac{\left(\bar{\gamma}^{*}\right)^{3 n}}{k^{2} M_{P}^{3} \sqrt{V_{0}} \alpha^{1 / 3}}\right)^{\frac{3}{4}}}\right)^{\frac{1}{3 \psi}} \times \\
& \left(\left[\sqrt{3} k M_{P} \sqrt{V_{0} \gamma^{*}}(-n)\left(N(2+\lambda)+\frac{k}{2}\right)\right]^{\frac{-3 \beta}{8+4 \lambda}}\right)^{\frac{1}{3 \psi}} \mathcal{P}_{s}^{\frac{1}{3 \psi}} .
\end{aligned}
$$

Besides, from Eqs.(14) and (15) the scalar spectral index and its running can be obtained as follow

$$
\begin{aligned}
n_{s}(n, k, N)= & \\
& 1+\frac{-24+k(6-12 n)+9 n}{2(-8(-3+n) N+k(4+n-4 N+2 n N))},
\end{aligned}
$$

and

$$
\begin{aligned}
\alpha_{s}(n, k, N)= & \\
& -\frac{3(-4(-3+n)+k(-2+n))(8-3 n+k(-2+4 n))}{(-8(-3+n) N+k(4+n-4 N+2 n N))^{2}} .
\end{aligned}
$$

From Eqs.(12) and (35), the tensor power spectrum in terms of the number of e-fold gives

$$
\begin{aligned}
& \mathcal{P}_{t}(n, k, N)=\frac{2 V_{0}}{3 \pi^{2} M_{P}^{4}} \times \\
& \left(\frac{\sqrt{3} k M_{P} \sqrt{V_{0}} \frac{\gamma^{*}(-n)}{\left.\gamma^{(}(2+\lambda)+\frac{k}{2}\right)}}{a^{\frac{4}{n+4}}}\right)^{\frac{k}{2+\lambda}},
\end{aligned}
$$

in addition, from Eqs. $(13,41)$ and $(37)$, the tensor to scalar ratio for strong regime is expressed as follows:

$$
\begin{aligned}
& r(n, k, N)=\frac{16 \sqrt{2} \times 3^{1 / 8} V_{0}}{25 M_{P}^{4}\left(\frac{\overline{\gamma^{*}}(3 n)}{k^{2} M_{P}^{3} \sqrt{V_{0}} \alpha^{1 / 3}}\right)^{3 / 4}} a^{\left(\frac{-4 k}{(n+4)(2+\lambda)}-3 \psi\right)} \times \\
& \left(\sqrt{3} k M_{P} \sqrt{V_{0}} \frac{\gamma^{*}}{\left.\gamma^{*}-n\right)}\left(N(2+\lambda)+\frac{k}{2}\right)\right)^{\frac{4 k-3 \beta}{8+4 \lambda}} .
\end{aligned}
$$


Now we should evaluate consistency of our model with observations originated from Planck 2013 and 2015 data $[1,2,4]$. Similar to the weak case, we can plot the regions in which a pair of our free parameters $(n, k)$ could satisfy the best concord between theory and observations for $r-n_{s}$ parameters. Such results can be found in figure 6, in which to fix our results we use the values $V_{0}=5.0 \times 10^{-20}$, and $M_{p}=1$. After that, by means of Eqs.(39) and (42) we can consider an arbitrary pair of free parameters as $(n=4, k=1)$ to investigate the results based on usual $r-n_{s}$ diagram. The investigation resulted in figure 7 and one can observe that our best fitted parameters are in a good agreement compared to Planck results [4]. Additionally, to investigate the behaviour of the running parameter we can use Eq.(40), and likelihood diagram from Planck results to compare our model, see figure 8.

Interestingly, our investigation confirms that although one of the best power law potentials to run the inflation is the quartic ones, another powers of power law potential could be considered as long as we choose the best fitted parameters in a correct way. So we can confirm that for both regimes the power law potentials in the framework of warm inflation are in good agreement compared to the planck results.

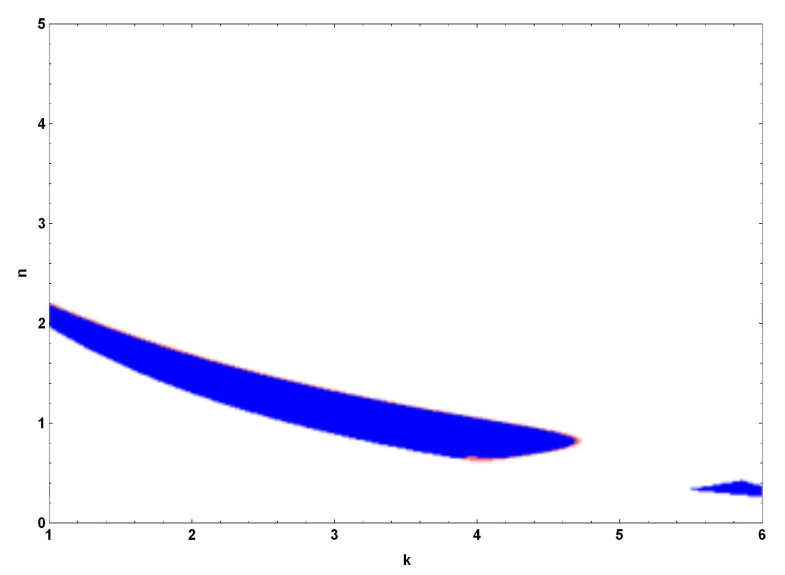

FIG. 6: This plot shows a set of $(n, k)$ pair that can give the best fitted values based on the $\left(r-n_{s}\right)$ diagram originated from Planck 2015 observational data. Here the dark blue color shows the results for $68 \%$ CLs of Planck 2015 data, and the light red color shows those, that put the result in $95 \%$ CLs. To plot this diagram we considered $V_{0}=5.0 \times 10^{-20}$, and $M_{p}=1$.

As explained for the weak regime, an important property of the warm inflationary paradigm is that the thermal fluctuations overcome the quantum fluctuations, since the fluid temperature is bigger than the Hubble parameter. To derive a healthy warm inflation, 

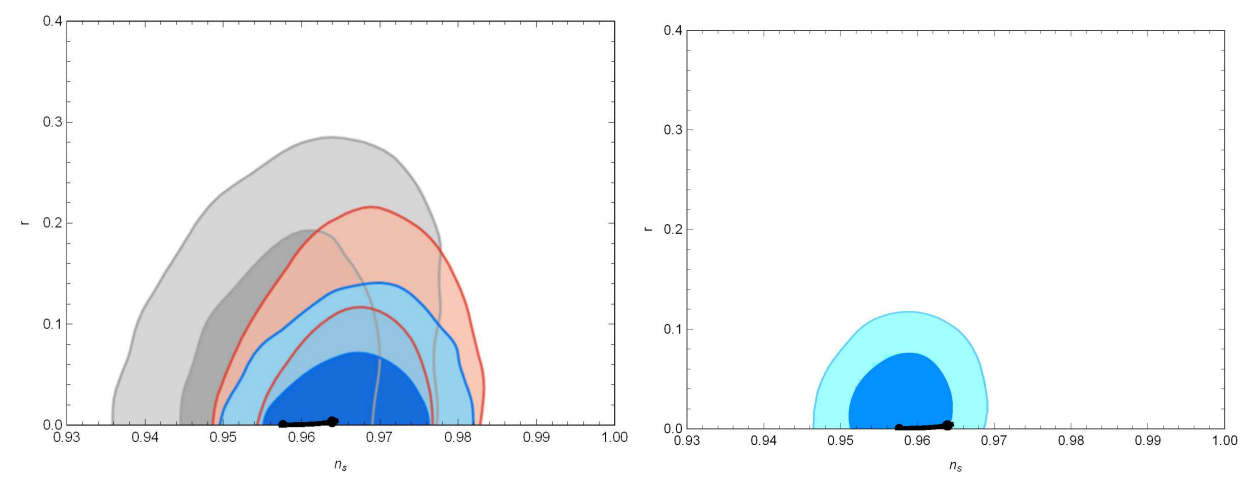

FIG. 7: The $r-n_{s}$ diagram shows Prediction of our theoretical results in strong regime for free parameters $\alpha=70, k=1, n=4$ and $V_{0}=5 \times 10^{-20}, M_{p}=1$ and $N_{e}=65$, in comparison to the observational data risen by Planck 2013, 2015 and 2018. In the left panel, the likelihood of Planck 2013 are indicated with grey contours, Planck TT+lowP with red contours, and Planck TT,TE,EE+lowP with blue contours. And in the right panel, the results of Planck 2018 are indicated by dark and light blue colours referring $68 \%$ and $95 \%$ confidence levels respectively. In both figures the thick black lines refers the predictions of theoretical results in which small and large circles are the values of $n_{s}$ at the number of e-folds $N=55, N=65$ respectively.

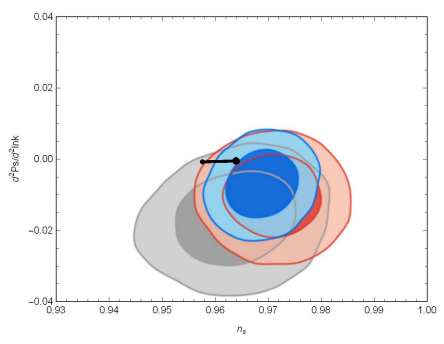

FIG. 8: This is the $d n_{s} / d N-n_{s}$ diagram which explains the running of the parameter $n_{s}$. In this figure the thick black line depicts the predictions of our model in which small and large circles are the value of $n_{s}$ at the number of e-fold $N=55$ and $N=65$ respectively. To plot this result we use the free parameters $k=1, n=4$, and $V_{0}=5 \times 10^{-20}, M_{p}=1, N_{e}=65$ and $\alpha=70$. In this diagram the grey contours indicated for likelihood of Planck 2013, Planck TT+lowP showed with red contours and the blue contours considered for Planck TT,TE,EE+lowP.y.

this condition should be justified during the primordial evolutions. Fig. 9 expresses the behavior of the ratio of the temperature to the Hubble parameter during such era. From the Figure 9, one can observe that the condition is satisfied during the inflationary phase of cosmological dynamics. 
In the strong regime it has assumed that $Q>1$, and it must control by means of the best fitted free parameters of the model. Therefore, one can see from the plot Fig. 10 that this condition is satisfied properly.

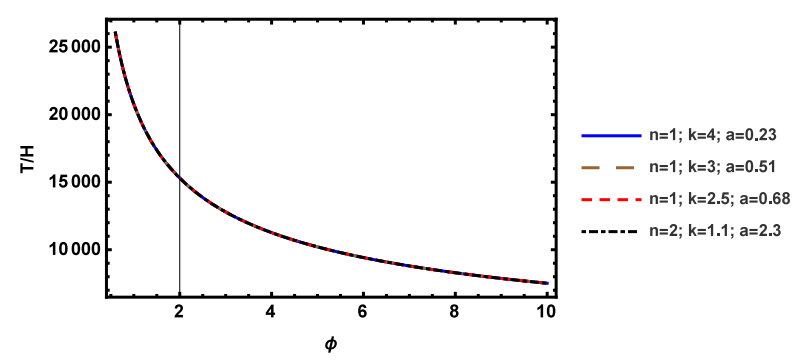

FIG. 9: The ratio of the temperature to the Hubble parameter during the inflationary period of the model in the weak dissipative regime versus the inflaton scalar field, $\phi$, for different values of $(n, k)$ and the parameter a. As one can see from the plots during inflation the temperature is larger than the Hubble parameter, and the condition $T>H$ is satisfied properly. To draw these figures we fixed the value of $V_{0}$ at $10^{-20}$.

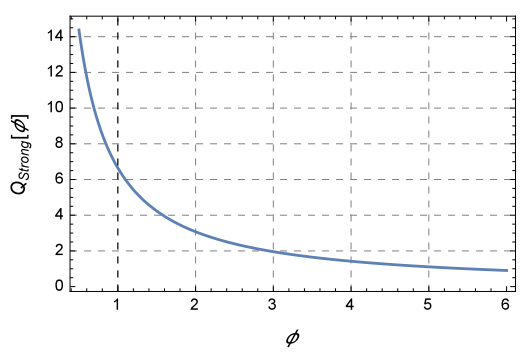

FIG. 10: This figure indicates the ratio of the dissipation parameter, $\Gamma$, to the Hubble parameter during the inflationary period of the model in the strong dissipative regime versus the inflaton scalar field, $\phi$, for the value of $(n=1.3, k=3)$ and the parameter $a=0.2$. As observed from the plot during inflation the parameter $\Gamma$ is large than the Hubble parameter, and the condition $Q=\Gamma / 3 H>1$ is satisfied properly. To draw these figure we fixed the value of $V_{0}$ at $10^{-20}$.

\section{CONCLUSIONS}

By introducing a general form for the power-law potentials and the dissipation coefficient in the frame work of warm inflationary scenario the constraints originated from Planck 2013 and 2015 have been studied. This study was separated into two well-know regimes, based on 
the friction of the primordial soup, namely weak and strong regimes. Besides, for a quasistatic universe the slow-roll constraints have been considered. After these simplification approaches, at first the different inflationary parameters such as power spectrum of scalar and tensor perturbations, scalar and tensor spectral indices, running of them and tensor-toscalar ratio, in both weak and strong regimes were been calculated. To visualize how the aforementioned parameters can satisfy a concord between theoretical and observations, we have considered a way in which a region of free parameters could do it, instead of just one set of the free parameters. In other word, by virtue of $r-n_{s}$ diagram, for both regimes, and fixing one of the free parameters the likelihood of free parameters $(n, k)$ were been plotted. The results have been appeared in figures 1 and 6 for weak and strong regimes respectively. In these figures two important CLs namely $68 \%$ and $95 \%$ for more clearance have been distorted by means of blue and red in colors. So we found the best fitted regions of free parameters that could make a good estimations for the theory. To examine our estimations an arbitrary pair of free parameters for both regimes have been illustrated. At first, and to avoid any confusions let us turn on to the weak regime investigations. For this regime the pair $(n=2, k=6)$ with $V_{0}=2.7 \times 10^{-25}$ have been selected. Based on this selection, the diagram $r-n_{s}$ and its running based on Planck data have been plotted, see figures 2 and 3. These figures have shown that our approach and also our results are in a good consistency in compared with the observations originated from Planck 2013 and 2015 data sets. Although, for weak regime we considered the power of variable of the potential as $k=6$, as well it could be observed for the quadratic or quartic powers there are no any failures. For the coefficient $V_{0}$ and other constants of the model our results can be justified compared to literature. We should add here, we can consider different values for the potential coefficient, i.e. $V_{0}$ in which the regions at figures 1 and 6 showon a little bit changes, so we avoided to the re-plotting them. Now we can turn our attention to the strong regime. In this regime because of the presence of the parameter $Q$, the situation is completely different and also maybe a little bit difficult comparing with weak case. For strong dissipation regime, the arbitrary pair of free parameters has been considered as $(n=1, k=4)$ with $V_{0}=5.0 \times 10^{-20}$. For this regime our freedom in selection of free parameter is more less than weak case, because of the smaller region for best fitted free parameters. Again, the accuracy of the obtained pairs for free parameters can be examined by means of the criterions arisen from Planck results. We did it by plotting the $r-n_{s}$ and its running and fortunately our results have been in a good 
agreement compared to the observations, Planck data. Our claims can be justified by looking at figures 7 and 8 for more investigations. We can continue this study about the relation between scalar field obtained here and also the scalar field obtained conformally to see the effects of amplitude of gravitational waves on the behaviour of inflationary observables, might in a new project. It has showed that Figs. 4 and 9 can justify the satisfactory behavior of the ratio of the temperature to the Hubble parameter during inflationary epoch for both weak and strong cases, i.e. $T>H$. To derive a healthy and self-consistent warm inflationary model, besides the whole above conditions one hast to control the constraints on the dissipation function for the both of the weak and the strong regimes. In doing so, we have plotted two figures which showed a satisfactory concord between theoretical results of this work and observational Planck data. The Figs. 5 and 10 have indicated the ratio of the dissipation parameter, $\Gamma$, to the Hubble parameter during the inflationary period of the model and were satisfied properly.

\section{ACKNOWLEDGEMENT}

The authors would like to thank A. Mohammadi for the serious discussions on the likelihood plots resulted in regional best fitted parameters. HS thanks A. Starobinsky for very constructive discussions about inflation during Helmholtz International Summer School 2019 in Russia. He grateful G. Ellis, A. Weltman, and UCT for arranging his short visit, and for enlightening discussions about cosmological fluctuations and perturbations for both large and local scales. He also thanks H. Firouzjahi for constructive discussions about inflation and perturbations. His special thanks go to his wife E. Avirdi for her patience during our stay in South Africa.

[1] P. A. R. Ade et al. (Planck Collaboration), Astron. Astrophys. 571, A1 (2014).

[2] P. A. R. Ade et al. (Planck Collaboration), Astron. Astrophys. 571, A22 (2014).

[3] P. A. R. Ade et al. (Planck Collaboration), Astron. Astrophys. 571, A24 (2014).

[4] P. A. R. Ade et al. (Planck Collaboration), Astron. Astrophys. 594, A20 (2016).

[5] Y. Akrami et al. (Planck Collaboration), arXiv:1807.06211 [astro-ph.CO].

[6] A. A. Starobinsky, Phys. Lett. B 91 , 99 (1980). 
[7] A. H. Guth, Phys. Rev. D 23, 347 (1981).

[8] A. D. Linde, Phys. Lett. B 108, 389 (1982).

[9] A. D. Linde Phys. Lett. B 129, 177 (1983).

[10] A. D. Linde Phys. Lett. B 175, 395 (1986).

[11] A. D. Linde Mod. Phys. Lett. A 1, 81 (1986).

[12] A. Albrecht, P. J. Steinhardt, Phys. Rev. Lett. 48 , 1220 (1982).

[13] A. R. Liddle, D. H. Lyth, Cosmological Inflation and Large-scale Structure, Cambridge University Press (2000).

[14] B. A. Bassett, S. Tsujikawa, and D. Wands, Rev. Mod. Phys. 78, 537 (2006).

[15] M. Lemoine, J. Martin, P. Peter, Inflationary Cosmology, Lect. Notes Phys. 738, Springer, Berlin Heidelberg (2008).

[16] W. H. Kinney, arXiv:0902.1529

[17] D. Baumann, arXiv:0907.5424 [hep-ph]

[18] D. Baumann, L. McAllister, arXiv:1404.2601 [hep-th]

[19] D. Larson, Astrophys. J. Suppl. 16,192 (2011).

[20] C. L. Bennett, et al., Astrophys. J. Suppl.192, 17 (2011).

[21] N. Jarosik, Astrophys. J. Suppl. 192, 14 (2011).

[22] A. R. Liddle, An introduction to cosmological inflation, astro-ph/9901124

[23] D. Langlois, hep-th/0405053

[24] D. H. Lyth, Phys. Rev. Lett. 78, 1861 (1997).

[25] D. H. Lyth, and A. Riotto, Phys. Rept. 314, 1 (1999).

[26] A. H. Guth, Phys. Rept. 333555 (2000).

[27] J. E. Lidsey, A. Liddle, et al.: Rev. Mod. Phys. 69, 373 (1997).

[28] V. F. Mukhanov, H. A. Feldman, and R. H. Brandenberger, Physics Reports A: Review Section of Physics Letters 215 , 203 (1992).

[29] H. Sheikhahmadi, S. Ghorbani, and Kh. Saaidi, Astrophys. Space Sci. 357, no. 2, 115 (2015).

[30] H. Sheikhahmadi, E. N. Saridakis, A. Aghamohammadid, and Kh. Saaidi, JCAP 10, 021 (2016).

[31] H. Sheikhahmadi, Eur. Phys. J. C 79, 451 (2019).

[32] R. H. Brandenberger, arXiv: hep-ph/9910410.

[33] A. N. Taylor. and A. Berera, Phys. Rev. D 62, 083517 (2000). 
[34] H. P. De Oliveira, S. E. Joras, Phys. Rev. D 64, 063513 (2001).

[35] H. P. De Oliveira, Phys. Lett. B 526, 1 (2002).

[36] A. Berera, and L. Z. Fang, Phys. Rev. Lett. 741912 (1995).

[37] L. M. H. Hall, I. G. Moss. and A. Berera, Phys. Rev. D 69, 083525 (2004).

[38] Bastero-Gil. M., Berera. A.: Phys. Rev. D 71, 063515 (2005).

[39] A. M. Cid, Phys. Lett. B 743, 127 (2015).

[40] L. Visinelli, JCAP 07, 054 (2016).

[41] A. M. Cid, S. del Campo, and R. Herrera, JCAP 10, 005 (2007).

[42] A. Berera, Phys. Rev. Lett. 75, 3218 (1995).

[43] A. Berera, Phys. Rev. D 54, 2519 (1996)

[44] A. Berera, Phys. Rev. D 55, 3346 (1997).

[45] A. Berera, Nucl. Phys. 585, 666 (2000).

[46] A. Berera, M. Gleiser, and R. O. Ramos, Phys. Rev. D 58123508 (1998).

[47] S. Rasouli, K. Rezazadeh, A. Abdolmaleki, and K. Karami, Eur. Phys. J. C 79, 79 (2019).

[48] L. Akhtari, A. Mohammadi, K. Sayar, and Kh. Saaidi, Astroparticle Physics, 90, 28 (2017).

[49] L. Z. Fang, Phys. Lett. B 95, 154 (1980).

[50] I. G. Moss, Phys. Lett. B 154, 120 (1985).

[51] R. Herrera, N. Videla, and M. Olivares, Eur. Phys. J. C 75, no. 5, 205 (2015);

[52] R. Herrera, M. Olivares, and N. Videla, Phys. Rev. D 90, no. 10, 103502 (2014);

[53] R. Herrera, M. Olivares, and N. Videla, Int. J. Mod. Phys. D 23, no. 10, 1450080 (2014).

[54] A. Berera, I. G. Mossm, and R. O. Ramos, Rept. Prog. Phys. 72, 026901 (2009).

[55] K. Sayar, A. Mohammadi, L. Akhtari, and Kh. Saaidi, Phys. Rev. D. 95, 023501 (2017).

[56] Y. Zhang, JCAP 0903, 023 (2009).

[57] M. Bastero-Gil. , A. Berera, and R. O. Ramos, JCAP 1107, 030 (2011).

[58] M. Bastero-Gil. , A. Berera, R. O. Ramos, and J. G. Rosa, JCAP 1301, 016 (2013).

[59] M. Bastero-Gil. , A. Berera, R. O. Ramos, and J. G. Rosa, JCAP 1410, no. 10, 053 (2014).

[60] I. G. Moss, C. Xiong, hep-ph/0603266

[61] G. Panotopoulos, N. Videlay, arXiv:1510.0698123 [gr-qc]

[62] Subhendra Mohanty and Akhilesh Nautiyal, Phys. Rev. D 78, 123515 (2008).

[63] L.Visinelli, JCAP 1109, 013 (2011). 
[64] L.Visinelli, JCAP 1501, 005 (2015).

[65] Y-y Wang et al., Phys.Rev. D 99, 10, 103529 (2019).

[66] S. Unnikrishnan, V. Sahni, JCAP 10, 063 (2013).

[67] S. del Campo, Warm inflationary universe models, in Aspects of Todays Cosmology, edited by A. Alfonso-Faus (InTech, Rijeka, Croatia, 2011).

[68] S. del Campo and R. Herrera, J. Cosmol. Astropart. Phys. 04 (2009) 005.

[69] L.Visinelli, JCAP 1607, 054 (2016)

[70] Mar Bastero-Gil, Arjun Berera, Rafael Hernandez-Jimenez, and Joao G. Rosa. Phys. Rev. D 99, 103520 (2019).

[71] Meysam Motaharfar, Vahid Kamali, Rudnei O. Ramos. Phys. Rev. D 99, 063513 (2019). 